Article

\title{
Amorphous InGaZnO Thin Film Transistor Fabricated with Printed Silver Salt Ink Source/Drain Electrodes
}

\author{
Caigui Yang ${ }^{1}$, Zhiqiang Fang ${ }^{1}$, Honglong Ning ${ }^{1, *}$ (i), Ruiqiang Tao ${ }^{1}$, Jianqiu Chen ${ }^{1}$, \\ Yicong Zhou ${ }^{1}$, Zeke Zheng ${ }^{1}$, Rihui Yao ${ }^{1, *}$ (D), Lei Wang ${ }^{1}$, Junbiao Peng ${ }^{1}$ and Yongsheng Song ${ }^{2}$ \\ 1 Institute of Polymer Optoelectronic Materials and Devices, State Key Laboratory of Luminescent Materials \\ and Devices, South China University of Technology, Guangzhou 510640, China; \\ 201520114097@mail.scut.edu.cn (C.Y.); fangzq1230@gmail.com (Z.F.); 201510102158@mail.scut.edu.cn (R.T.); \\ c.jianqiu@mail.scut.edu.cn (J.C.); zhou.yicong@mail.scut.edu.cn (Y.Z.); 201520114219@mail.scut.edu.cn (Z.Z.); \\ mslwang@scut.edu.cn (L.W.); psjbpeng@scut.edu.cn (J.P.) \\ 2 Guangdong Fenghua Advanced Technology Holding Co., Ltd., Zhaoqing 526060, China; \\ xmub@163.com \\ * Correspondence: ninghl@scut.edu.cn (H.N.); yaorihui@scut.edu.cn (R.Y.)
}

Received: 25 June 2017; Accepted: 14 August 2017; Published: 16 August 2017

\begin{abstract}
Recently, amorphous indium-gallium-zinc-oxide thin film transistors (a-IGZO TFTs) with inkjet printing silver source/drain electrodes have attracted great attention, especially for large area and flexible electronics applications. The silver ink could be divided into two types: one is based on silver nanoparticles, and the other is silver salt ink. Organic materials are essential in the formulation of nanoparticle ink as a strong disperse stabilizer to prevent agglomeration of silver particles, but will introduce contact problems between the silver electrodes and the a-IGZO active layer after annealing, which is difficult to eliminate and leads to poor device properties. Our experiment is aimed to reduce this effect by using a silver salt ink without stabilizer component. With optimized inkjet printing conditions, the high performance of a-IGZO TFT was obtained with a mobility of $4.28 \mathrm{~cm}^{2} / \mathrm{V} \cdot \mathrm{s}$ and an on/off current ratio over $10^{6}$. The results have demonstrated a significant improvement for a-IGZO TFTs with directly printed silver electrodes. This work presents a promising platform for future printed electronic applications.
\end{abstract}

Keywords: a-IGZO TFTs; inkjet printing; source/drain electrodes; silver salt ink

\section{Introduction}

Thin film transistors (TFTs) are now fundamental electronic components in virtually all consumer and professional display products, from smart phones to flat panel TVs [1-3]. With the extensive application of large area and flexible electronics, inkjet printing technology for TFTs has become a hot spot benefitting from not requiring a conventional vacuum process and enabling us to reduce the fabrication costs [4-6]. Conductive ink, as the core of printing electronic technology, has an important impact on the performance of device. Silver possesses excellent conductivity and anti-oxidation properties, thus silver based inks show a potential application in fabrication of high performance electrodes of TFT by inkjet printing [7-10]. Silver based ink could be divided into two types: one is based on silver nanoparticles [11-13] and the other is silver salt ink [14-16]. Organic polymers as a disperse stabilizer are inevitably presented in nanoparticle inks to prevent aggregation of silver nanoparticles [13], and can be usually removed by high temperature sintering or high-intensity light curing [17], to facilitate the merging of silver nanoparticles. In contrast, the silver salt could be stably dissolved in the solvent without the disperse stabilizer [15], and could be thermally self-reduced to metallic silver at low temperatures [18,19]. 
Amorphous indium-gallium-zinc-oxide thin film transistors (a-IGZO TFTs) with inkjet printed silver source/drain (S/D) electrodes have been proposed in many reports [20-23]. The experimental conclusions show a contact problem between the silver S/D electrodes and the a-IGZO active layer when using silver nanoparticles ink. The organic carbon residues are difficult to remove after the annealing process, which results in poor device performance that cannot meet the requirements of driving large-sized flat panel displays [24]. Therefore, the development of electronics fabricated with silver salt ink are of great potential in display application.

In this work, a-IGZO TFTs with printed S/D electrodes are fabricated using a silver salt ink and silver nanoparticle ink respectively. It was found that the organic carbon could be effectively reduced at the interface by using silver salt ink, which indicates the solving of the contact problem to some extent by this manner, accordingly well-performing a-IGZO TFTs were obtained successfully and reasonably.

\section{Experimental}

The schematic structure of the bottom-gate, top-contact a-IGZO TFT with inkjet print Ag S/D electrodes is shown in Figure 1a. To prepare the device, a 300-nm-thick layer of Al:Nd alloy (3 wt \% of $\mathrm{Nd}$ ) was firstly sputtered on glass as gate electrode at room temperature. Then, the film was anodized in the electrolyte consisting of ammonium tartrate solution and ethylene glycol, accordingly forming a 200-nm-thick $\mathrm{Al}_{2} \mathrm{O}_{3}: \mathrm{Nd}$ gate dielectric layer on the surface of $\mathrm{Al}$ gate. Next, a 25-nm-thick a-IGZO active layer was deposited on $\mathrm{Al}_{2} \mathrm{O}_{3}$ by radio frequency (RF) magnetron sputtering in an $\mathrm{Ar} / \mathrm{O}_{2}$ gas mixture with a power of $80 \mathrm{~W}$, and subsequently the sample was annealed at $400{ }^{\circ} \mathrm{C}$ for $1 \mathrm{~h}$ on a hotplate. Finally, the Ag S/D electrodes were directly printed onto the a-IGZO active layer.
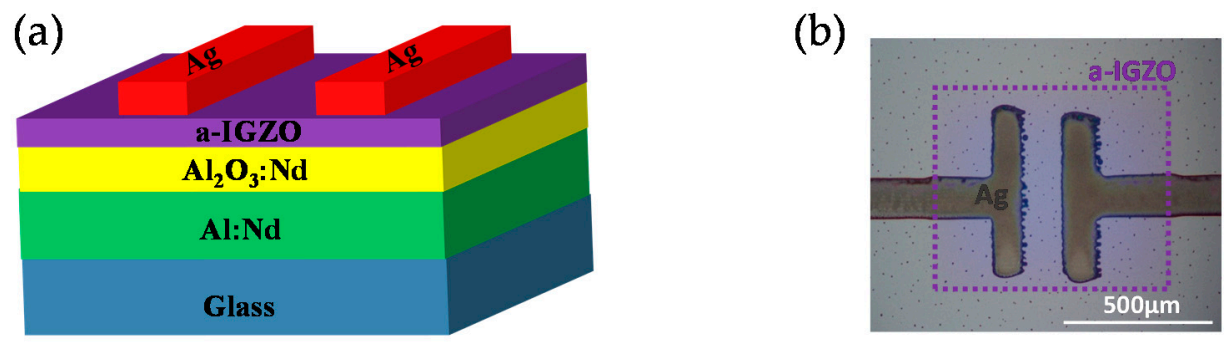

Figure 1. (a) Schematic of a-IGZO TFT with inkjet printing Ag S/D electrodes; (b) Optical microscope image of device finally fabricated.

For the formation of top Ag S/D, a purely transparent commercial ink (TEC-IJ-010, INKTEC, Ansan-city, Kyungki-do, Korea), which consists of silver salt and ethanol-based solvent, is not formulated by nanoparticle structure and thus could be stably dissolved in the solvent without disperse stabilizer. It contains $15 \mathrm{wt} \%$ silver contents with a viscosity of 9-15 cps and a surface tension of 30-32 dynes $/ \mathrm{cm}$. The silver ink was patterned onto the a-IGZO via a piezoelectric inkjet printer (DMP2800, Fujifilm Dimatix, Santa Clara, CA, USA) installed with a 1 pL cartridge. An optimized waveform was set with a drop velocity of $5 \mathrm{~m} / \mathrm{s}$. The temperature of the cartridge and substrate were maintained at $60{ }^{\circ} \mathrm{C}$ during the printing process, meanwhile, droplets were deposited with a spacing of $25 \mu \mathrm{m}$ to obtain uniform Ag S/D electrodes on a-IGZO as shown in Figure 1b. Channel length/width measurement was 550/154 $\mu \mathrm{m}$. After printing, the device was sintered at $150{ }^{\circ} \mathrm{C}$ for 10 min on hotplate. Comparing devices fabricated with different types silver inks, the device with printed Ag S/D electrodes using a silver nanoparticle ink (30-35\% in volume, DGP 40TE-20C, Advanced Nano Products, Bugang-myeon, Sejong-Si, Korea) was further fabricated under optimized conditions. The droplet ejection velocity was $2 \mathrm{~m} / \mathrm{s}$, the substrate temperature was $60^{\circ} \mathrm{C}$, and the interdrop space was $35 \mu \mathrm{m}$. After printing, the Ag electrodes were dried with UV equipment (Intelli-Ray 600, Uvitron, West Springfield, MA, USA) using 100\% intensity for $300 \mathrm{~s}$. Channel length/width measurement was $549 / 142 \mu \mathrm{m}$. 
The size of channel was measured with a polarized optical microscope (Nikon, DeWitt, IA, USA). The structure of Ag film on glass substrate was obtained by scanning electron microscopy, scanning electron microscopy (SEM, NOVA NANOSEM 430, FEI, Eindhoven, The Netherlands), and X-ray diffraction (XRD, Empyrean, PANalytical, Almelo, The Netherlands). The electrical properties of devices were characterized in ambient air using a semiconductor parameter analyzer (Agilent 4155C, Santa Clara, CA, USA). The properties of the Ag/a-IGZO interface were analyzed by transmission electron microscope, (TEM, Bruker, Adlershof, Berlin, Germany) with an electron energy loss spectroscope (EELS, Gatan Enfinium ER Model 977, Pleasanton, CA, USA).

\section{Results and Discussion}

Figure 2 shows the film morphology of the inkjet printing two types silver inks on a-IGZO/ $\mathrm{Al}_{2} \mathrm{O}_{3}$ at different substrate temperature (Supporting Information Figure S10). For silver nanoparticle ink, the evaporation rate of the droplets near the periphery area is larger than that at the center, accordingly creating a capillary flow from the center to the edge of the droplets, and carrying the suspended particles to the edge. As the substrate temperature increases, the effect was promoted and the film near the periphery area exhibited a shiny and silver appearance at $60^{\circ} \mathrm{C}$ as shown in Figure 2a. For silver salt ink as shown in Figure 2b, the solutes migrated to the edge of the film at room temperature, thus the a-IGZO under layer at the central area is exposed. As the the substrate temperature increased, the solvent evaporation was accelerated, and the edges of the droplets were pinned on the substrate surface. At $40{ }^{\circ} \mathrm{C}$, the film quality was improved, and uniform film was finally formed at $60{ }^{\circ} \mathrm{C}$. To make the silver ink solvent vaporize quickly, the substrate temperature was defined at $60^{\circ} \mathrm{C}$.

(a)

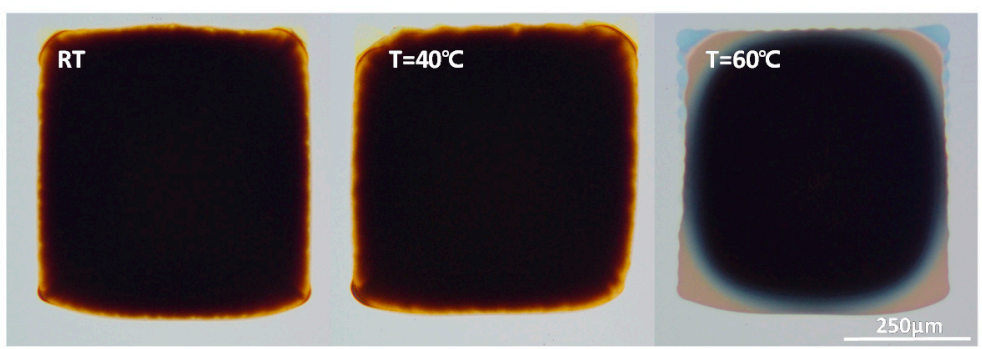

(b)

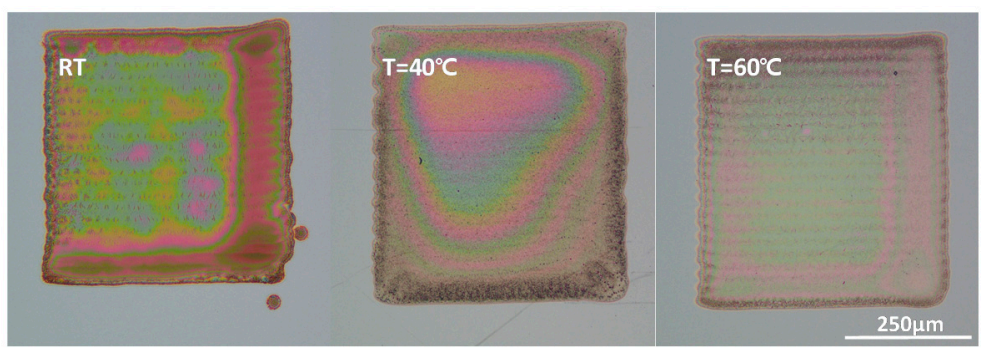

Figure 2. Inkjet printed with (a) silver nanoparticles ink film and (b) silver salt ink film on a-IGZO $/ \mathrm{Al}_{2} \mathrm{O}_{3}$ with substrate temperatures of $\mathrm{RT}, 40^{\circ} \mathrm{C}$ and $60{ }^{\circ} \mathrm{C}$, respectively.

To further investigate the microscale differences of two kinds of printed silver electrodes, a SEM measurement was adopted. For silver nanoparticle ink, Figure 3a shows Ag particles randomly merged together after heat treatment, the overall density of the Ag electrodes is pretty good, but a number of boundaries exist between the silver particles. Comparatively, for silver salt ink, Figure 3b clearly shows that the electrode consisting of interconnected Ag particles and voids, which is demonstrated to be a typical phenomenon using silver salt ink. It is well known that the solvent evaporates quickly and the silver salt is reduced to silver nanoparticles during curing process, and eventually forms a conductive silver film with pores [25]. The XRD patterns in Figure 3c demonstrate that the two types 
of Ag electrodes have characteristic peaks of metallic pure silver with a good crystallization after thermal treatment.

(a)

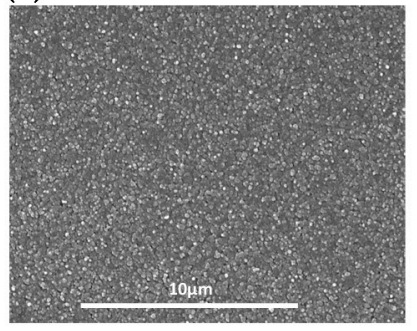

(b)

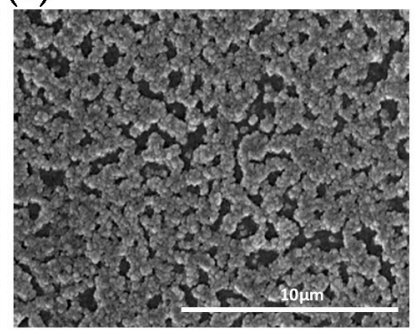

(c)

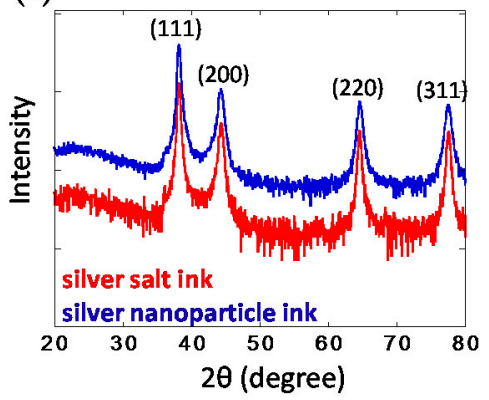

Figure 3. SEM images of the inkjet printed silver electrodes of TFTs by using (a) silver nanoparticles ink and (b) silver salt ink, respectively; (c) XRD patterns of the silver films after thermal treatment.

Figure $4 \mathrm{a}, \mathrm{b}$ respectively show the output and transfer characteristics for the device by using silver nanoparticles ink (Supporting Information Figure S11). The device exhibits a poor electrical performance, which presents a mobility $0.15 \mathrm{~cm}^{2} / \mathrm{V} \cdot \mathrm{s}$, on / off current ratio of over $10^{4}$, and turn-on voltage of about $6.1 \mathrm{~V}$. Furthermore, the output curve shows unstable and low on current value, which decreases at high drain voltage bias and suggests the prevalence of charge traps. Comparatively, Figure $4 \mathrm{c}, \mathrm{d}$ respectively show the output and transfer characteristics for the device by using silver salt ink. The device exhibits excellent field effect transistor characteristics, where the output curve shows a good saturation behavior. This device presents a higher mobility of $4.28 \mathrm{~cm}^{2} / \mathrm{V} \cdot \mathrm{s}$, a higher on/off current ratio of over $10^{6}$, and a lower turn-on voltage close to zero.

(a)
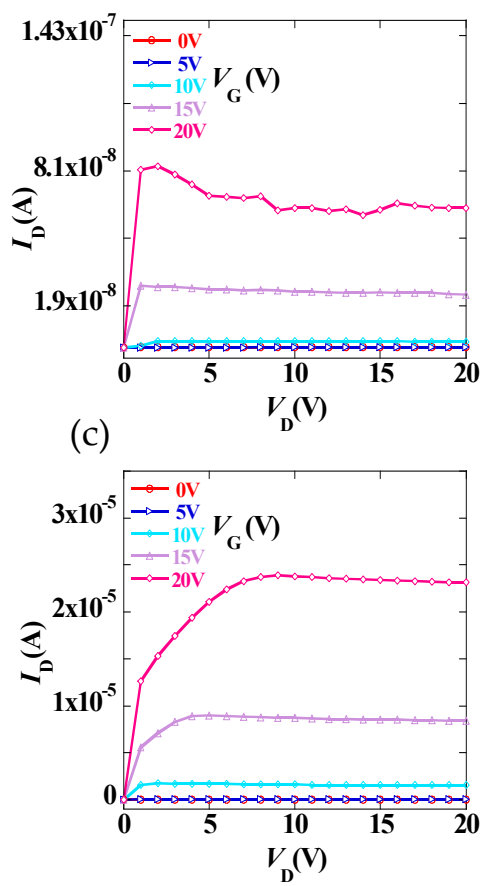

(b)

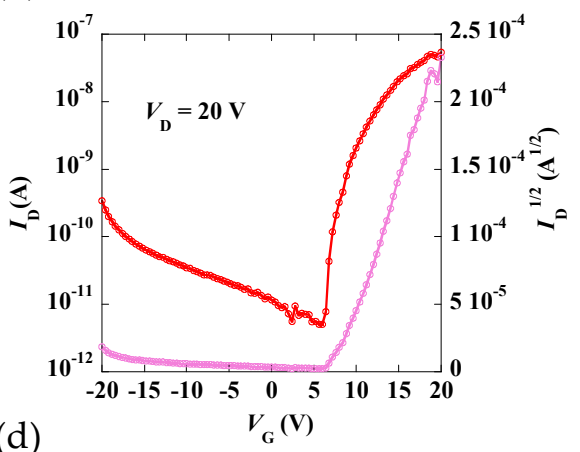

(d)

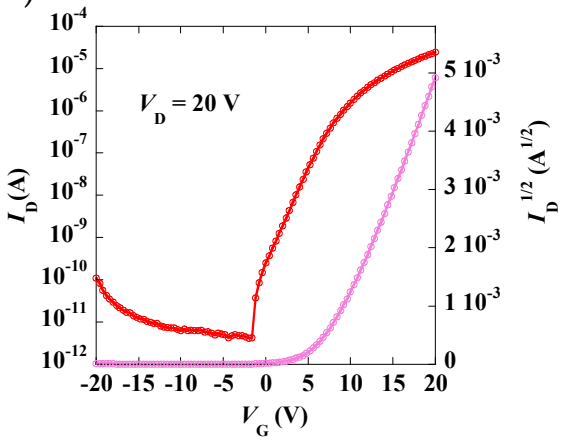

Figure 4. (a) Output and (b) transfer characteristics of the device with silver nanoparticle ink; (c) Output and (d) transfer characteristics of the device with silver salt ink. 
TEM and EELS measurements were used to observe the Ag/a-IGZO interface and detect the distribution of elements of silver, carbon, and oxygen, and the result is shown in Figure 5. Figure 5a presents the TEM images of the Ag/a-IGZO structure fabricated using the silver salt ink, and the amplified area of the interface is shown in Figure 5b. By ELLS measurements of the interface along the scan line, no phase separation was observed at the interface, which indicates the solvent in silver salt ink without disperse stabilizer evaporated thoroughly, and was illustrated in Figure 5c. The same measurements were taken to detect the interface using the sliver nanoparticles ink, and the results are shown in Figure 5d-f. Compared to the silver salt ink, the interface using the silver nanoparticles ink presents a clearly carbon phase accumulation, which reveals the existence of an organic layer of the disperse stabilizer residues that blocks the transportation of electrons [21]. As described above, a better contact between Ag electrodes and a-IGZO was proved for the device fabricated with silver salt ink. The higher performances of the device fabricated with silver salt ink could be ascribed to the better contact between the Ag S/D electrodes and a-IGZO.

(a)

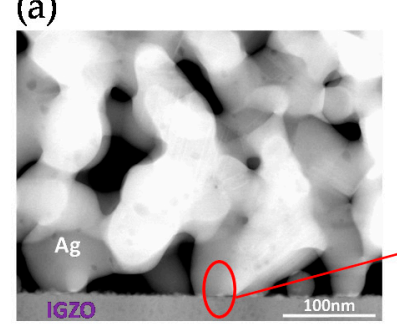

(d)

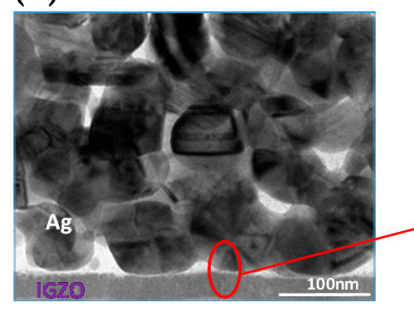

(b)

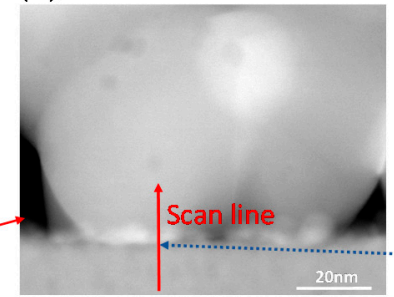

(e) (c)

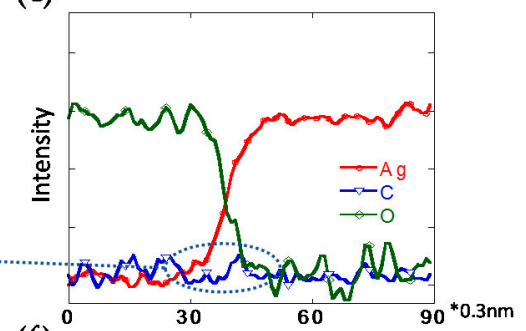

(f)

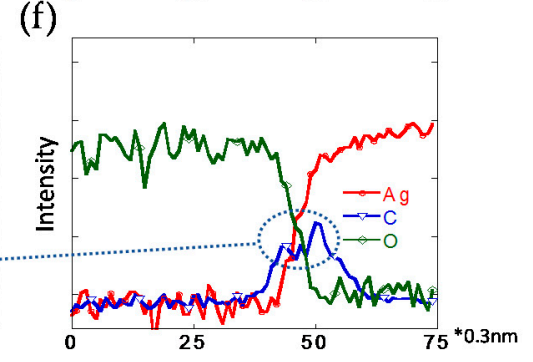

Figure 5. Comparision of Ag/a-IGZO interfaces for silver salt and nanoparticles inks. (a) TEM image of the silver/a-IGZO structure for silver salt ink; $(\mathbf{b}, \mathbf{c})$ Respectively show the amplified area and the EELS measurement of $\mathrm{Ag}, \mathrm{C}$, and $\mathrm{O}$ along marked scan line; (d-f) TEM images and the ELLS measurement for the nanoparticles ink.

\section{Conclusions}

The a-IGZO TFTs with printed Ag S/D electrodes from different types of silver inks were studied in this work under optimized conditions. The performance of devices fabricated with silver salt ink was demonstrated much better characteristics than those fabricated with silver nanoparticle ink. Almost no carbon remained at the interface using the silver salt ink, which benefits from the easily removed solvent without adding any organic disperse stabilizer, thus a good contact between Ag electrodes and a-IGZO was formed. As a result, a reasonably well performing a-IGZO TFT was obtained with a mobility of $4.28 \mathrm{~cm}^{2} / \mathrm{V} \cdot \mathrm{s}$ and a current on/off ratio over $10^{6}$. This work provides a promising route for future printed electronic applications.

Supplementary Materials: The following are available online at http:/ /www.mdpi.com/2076-3417/7/8/844/s1. Figure S1. Images of the inkjet printing silver ink on (a) the glass and (b) the Al2O3; Figure S2. The thickness of the Ag electrodes by using two types of silver ink; Figure S3. Transfer characteristics curves (ID-VG) for devices by using (a) silver salt ink and (b) silver nanoparticle ink; Figure S4. Transfer characteristics curves (ID-VG) for devices by using silver nanoparticle ink under (a) thermal annealing and (b) UV-curing; Figure S5. Schematic diagram of circuit for thin film transistor; Figure S6. The depth profile of the devices by using (a) silver nanoparticle ink; (b) EDS mapping; and (c) line scanning of the Ag/a-IGZO interface; Figure S7. The contact resistance of 
devices with (a) silver salt ink and (b) silver nanoparticle ink; Figure S8. The variations of time-dependent transfer property under $(\mathbf{a})$ positive gate-bias-stress $(\mathrm{VG}=10 \mathrm{~V})$ and $(\mathbf{b})$ negative gate-bias stress $(\mathrm{VG}=-10 \mathrm{~V})$; Figure S9. The mobility and the density of the a-IGZO by the Hall measurements; Figure S10. Inkjet printing silver nanoparticle ink film on (a) glass and (b) a-IGZO/Al2O3 with substrate temperature of RT, $40^{\circ} \mathrm{C}$ and $60{ }^{\circ} \mathrm{C}$, respectively; Inkjet printing silver salt ink film on (c) glass and (d) a-IGZO/Al2O3 with substrate temperature of $\mathrm{RT}, 40^{\circ} \mathrm{C}$ and $60^{\circ} \mathrm{C}$, respectively; Figure S11. The output characteristic curves (ID-VD) and transfer characteristic curves (ID-VG) of the devices express through different greyscale.

Acknowledgments: This work was supported by National Key R \& D Program of China (No. 2016YFB0401504), National Key Basic Research, NSFC (No. U1601651) and Development Program of China (973 program, Grant No. 2015CB655004) founded by MOST, Guangdong Natural Science Foundation (2016A030313459), Science and Technology Project of Guangdong Province (2014B090915004, 2015A010101323, and 2016B090907001), the Fundamental Research Funds for the Central Universities (2015ZP024 and 2015ZZ063). The authors are grateful to SAE Magnetics (Dongguan, Guangdong) LTD for assistance in FIB-TEM characterization.

Author Contributions: Honglong Ning, Rihui Yao, Caigui Yang, Ruiqiang Tao and Zhiqiang Fang designed the research; Caigui yang and Jianqiu Chen performed the experiments; Zeke Zheng, Jianqiu Chen and Yicong Zhou analyzed the data; Ruihui Yao, Lei Wang, Junbiao Peng and Yongsheng Song provided valuable discussions and suggestions; Honglong Ning, Caigui Yang and Ruiqiang Tao wrote the paper.

Conflicts of Interest: The authors declare no conflict of interest.

\section{References}

1. Street, R.A. Thin-Film Transistors. Adv. Mater. 2009, 21, 2007-2022. [CrossRef]

2. Ito, M.; Miyazaki, C.; Ishizaki, M.; Kon, M.; Ikeda, N.; Okubo, T.; Matsubara, R.; Hatta, K.; Ugajin, Y.; Sekine, N. Application of amorphous oxide TFT to electrophoretic display. J. Non-Cryst. Solids 2008, 354, 2777-2782. [CrossRef]

3. Mo, Y.G.; Kim, M.; Kang, C.K.; Jeong, J.H.; Park, Y.S.; Choi, C.G.; Kim, H.D.; Kim, S.S. Amorphous-oxide TFT backplane for large-sized AMOLED TVs. J. Soc. Inf. Disp. 2012, 19, 16-20. [CrossRef]

4. Subramanian, V.; Chang, J.B.; de la Fuente Vornbrock, A.; Huang, D.C. Printed electronics for low-cost electronic systems: technology status and application development. In Proceedings of the ESSDERC 2008 38th European Solid-State Device Research Conference, Edinburgh, UK, 15-19 September 2008; pp. 17-24.

5. Kim, D.; Jeong, S.; Lee, S.; Park, B.K.; Moon, J. Organic thin film transistor using silver electrodes by the ink-jet printing technology. Thin Solid Films 2007, 515, 7692-7696. [CrossRef]

6. Sowade, E.; Mitra, K.Y.; Ramon, E.; Martinez-Domingo, C.; Villani, F.; Loffredo, F.; Gomes, H.L.; Baumann, R.R. Up-scaling of the manufacturing of all-inkjet-printed organic thin-film transistors: Device performance and manufacturing yield of transistor arrays. Org. Electron. 2016, 30, 237-246. [CrossRef]

7. Tao, R.; Ning, H.; Fang, Z.; Chen, J.; Cai, W.; Zhou, Y.; Zhu, Z.; Yao, R.; Peng, J. Homogeneous Surface Profiles of Inkjet-Printed Silver Nanoparticle Films by Regulating Their Drying Microenvironment. J. Phys. Chem. C 2017, 121, 8992-8998. [CrossRef]

8. Tang, W.; Feng, L.; Zhao, J.; Cui, Q.; Chen, S.; Guo, X. Inkjet printed fine silver electrodes for all-solution-processed low-voltage organic thin film transistors. J. Mater. Chem. C 2014, 2, 1995-2000. [CrossRef]

9. Teng, K.; Vest, R. Liquid Ink Jet Printing with MOD Inks for Hybrid Microcircuits. IEEE Trans. Compon. Hybrids Manuf. Technol. 1987, 10, 545-549. [CrossRef]

10. Gamerith, S.; Klug, A.; Scheiber, H.; Scherf, U.; Moderegger, E.; List, E.J.W. Direct Ink-Jet Printing of Ag-Cu Nanoparticle and Ag-Precursor Based Electrodes for OFET Applications. Adv. Funct. Mater. 2007, 17, 3111-3118. [CrossRef]

11. Vaseem, M.; Lee, K.M.; Hong, A.; Hahn, Y.B. Inkjet Printed Fractal-Connected Electrodes with Silver Nanoparticle Ink. ACS Appl. Mater. Interface 2012, 4, 3300-3307. [CrossRef] [PubMed]

12. Lee, H.; Chou, K.; Huang, K. Inkjet printing of nanosized silver colloids. Nanotechnology 2005, 16, $2436-2441$. [CrossRef] [PubMed]

13. Sondi, I.; Goia, D.V.; Matijevi, E. Preparation of highly concentrated stable dispersions of uniform silver nanoparticles. J. Colloid Interface Sci. 2003, 260, 75-81. [CrossRef]

14. Nie, X.; Wang, H.; Zou, J. Inkjet printing of silver citrate conductive ink on PET substrate. Appl. Surf. Sci. 2012, 261, 554-560. [CrossRef] 
15. Jahn, S.F.; Blaudeck, T.; Baumann, R.R.; Jakob, A.; Ecorchard, P.; Rüffer, T.; Lang, H.; Schmidt, P. Inkjet Printing of Conductive Silver Patterns by Using the First Aqueous Particle-Free MOD Ink without Additional Stabilizing Ligands. Chem. Mater. 2010, 22, 3067-3071. [CrossRef]

16. Wu, Y.; Li, Y.; Ong, B.S. A Simple and Efficient Approach to a Printable Silver Conductor for Printed Electronics. J. Am. Chem. Soc. 2007, 129, 1862-1863. [CrossRef] [PubMed]

17. Sowade, E.; Kang, H.; Mitra, K.Y.; Weiß, O.J.; Weber, J.; Baumann, R.R. Correction: Roll-to-roll infrared (IR) drying and sintering of an inkjet-printed silver nanoparticle ink within 1 second. J. Mater. Chem. C 2015, 3, 11974. [CrossRef]

18. Dearden, A.L.; Smith, P.J.; Shin, D.; Reis, N.; Derby, B.; O’Brien, P. A Low Curing Temperature Silver Ink for Use in Ink-Jet Printing and Subsequent Production of Conductive Tracks. Macromol. Rapid Commun. 2005, 26, 315-318. [CrossRef]

19. Smith, P.J.; Shin, D.Y.; Stringer, J.E.; Derby, B.; Reis, N. Direct ink-jet printing and low temperature conversion of conductive silver patterns. J. Mater. Sci. 2006, 41, 4153-4158. [CrossRef]

20. Ueoka, Y.; Nishibayashi, T.; Ishikawa, Y.; Yamazaki, H.; Osada, Y.; Horita, M.; Uraoka, Y. Analysis of printed silver electrode on amorphous indium gallium zinc oxide. Jpn. J. Appl. Phys. 2014, 53, 3E-4E. [CrossRef]

21. Ning, H.; Chen, J.; Fang, Z.; Tao, R.; Cai, W.; Yao, R.; Hu, S.; Zhu, Z.; Zhou, Y.; Yang, C.; et al. Direct Inkjet Printing of Silver Source/Drain Electrodes on an Amorphous InGaZnO Layer for Thin-Film Transistors. Materials 2017, 10, 51. [CrossRef] [PubMed]

22. Secor, E.B.; Smith, J.; Marks, T.J.; Hersam, M.C. High-Performance Inkjet-Printed Indium-Gallium-Zinc-Oxide Transistors Enabled by Embedded, Chemically Stable Graphene Electrodes. ACS Appl. Mater. Interface 2016, 8, 17428-17434. [CrossRef] [PubMed]

23. Ueoka, Y.; Ishikawa, Y.; Bermundo, J.; Yamazaki, H.; Urakawa, S.; Osada, Y.; Horita, M.; Uraoka, Y. Effect of contact material on amorphous InGaZnO thin-film transistor characteristics. Jpn. J. Appl. Phys. 2014, 53, 3C-4C. [CrossRef]

24. Yabuta, H.; Sano, M.; Abe, K.; Aiba, T.; Den, T.; Kumomi, H.; Nomura, K.; Kamiya, T.; Hosono, H. High-mobility thin-film transistor with amorphous $\mathrm{InGaZnO} 4$ channel fabricated by room temperature rf-magnetron sputtering. Appl. Phys. Lett. 2006, 89, 112123. [CrossRef]

25. Dong, Y.; Li, X.; Liu, S.; Zhu, Q.; Li, J.; Sun, X. Facile synthesis of high silver content MOD ink by using silver oxalate precursor for inkjet printing applications. Thin Solid Films 2015, 589, 381-387. [CrossRef] 